\title{
Tratamento de pneumonia em pacientes hospitalizados - resultado de um estudo clínico multicêntrico utilizando uma cefal osporina de quarta geração (cefepima)
}

\author{
E. A. S. DE MEDEIROS
}

Disciplina de Doenças I nfecciosas eParasitárias da U niversidadeF ederal deSão Pauloe da Divisão de M oléstias I nfecciosas do H ospital das Clínicas - Faculdade de Medicina da Universidade de São Paulo, São Paulo, SP.

RESUMO - Oвj etıvo. Avaliar a eficácia e a segurança da cefepima no tratamento de pneumonia grave em pacientes hospitalizados.

Casuística e Métodos. Realizamos um estudo perspectivo, multicêntrico, não comparativo envolvendo 148 pacientes ( 62 com pneumonia hospitalar, 34 com pneumonia comunitária e 52 formas indefinidas). A cefepima foi administrada por via intravenosa (1.000 a $2.000 \mathrm{mg}$ cada 12 horas), sendo que as doses também foram ajustadas para a função renal. A resposta clínica foi avaliada 48 horas após o térmi no da terapêutica com cefepima.

Resultados. A média de idade foi de 56,4 $\pm 20,31$ anos. As bactérias mais comuns isoladas nos pacientes com pneumonia hospitalar foram: 5 Pseudomonas aeruginosa (8,06\%); 7 Pseudomonas sp. (11,29\%); 6 Klebsiella sp. (9,68\%); 3 E. coli $(4,84 \%) ; 2$ Acinetobacter baumanni $(3,23 \%) ; 3$

\section{INTRODUÇÃO}

O termo pneumonia refere-se a infecção do trato respiratório inferior que primariamente envolve o pulmão. As pneumonias podem ser divididas em comunitárias e hospitalares. Esta classificação é baseada no ambiente em que a infecção é adquiri da e apresentam agentes etiológicos, fatores de risco e evol ução clínica diferentes.

A pneumonia adquirida na comunidade permanece como importante causa de hospital ização e de morte. Estima-se que entre duas a $15 / 1.000$ pessoas a cada ano adquirem pneumonia e cerca de 20 a $40 \%$ necessitam ser hospitalizadas sendo que de 5 a $30 \%$ requerem tratamento em unidades de terapia intensiva. A mortalidade de pneumonia adquirida na comunidade antes da antibioticoterapia era de 200/100.000 pessoas por ano. Com a introdução da penicilina, em 1940, diminuiu para cerca de 70/100.000 pessoas por ano. ${ }^{1,2}$

E $m$ um estudo real izado em 1964 foi observado uma taxa de letalidade de $25 \%$ em uma série de pacientes que desenvolveram pneumonia com
Staphylococcus aureus (4,84\%); 3 Streptococcus pneumoniae $(4,84 \%)$; 5 outras $(8,06 \%)$. Os mais comuns isolados nos pacientes com pneumonia adquirida na comunidade foram: 2 Streptococcus pneumoniae $(5,88 \%)$; 1 S. aureus $(2,94 \%) ; 2$. aeruginosa $(5,88 \%)$ e $2 \mathrm{~K}$. pneumoniae $(5,88 \%)$. A eficácia clínica foi observada em 137/148 dos casos $(92,56 \%)$ sendo que a resolução parcial foi obtida em $20,27 \%$ e cura em $72,29 \%$. Falha de tratamento foi encontrado em 10 pacientes (6,75\%) e um caso não foi avaliado. E ventos adversos foram observados em $5 / 148$ pacientes $(3,38 \%$ ).

Conclusão. Nosso estudo sugere que a cefepima é seguro e efetivo no tratamento de pneumonia grave em pacientes hospitalizados.

UNITERMOS: Pneumonia adquirida na comunidade. Pneumonia nosocomial. Cefalosporina. Cefepima.

bacteremia, indicando que a antibioticoterapia ainda possui grandes limitações. ${ }^{3}$

As pneumonias hospital ares são consideradas a principal infecção adquirida em hospitais brasil eiros sendo responsável por 13 a 18\% de todas infecções hospitalares. Em São Paulo - Brasil - em diversas instituições tais como Hospital das Clíni cas da Universidade de São Paulo, H ospital São Paul o da E scola Paulista de Medicina, UNIFESP e Hospital do Servidor Público E stadual, a pneumonia hospitalar está entre as principais infecções, sendo mais freqüente em unidades de terapia intensiva. Medeiros (1991) em um estudo controlado, realizado na U nidade de Terapia Intensiva do Hospital São Paulo da Escola Paulista de Medicina, UNIFESP, analisou 60 episódios consecutivos de pneumonia hospitalar sendo que a taxa de letalidade dos casos foi de $53,3 \%$ enquanto a dos controles de $28,3 \%$. A letalidade atribuída foi de $25 \%$ com intervalo de confiança (IC $=95 \%$ ) de 7,3 a $42 \%$, risco relativo de $1,88, \mathrm{IC}=95 \%$, de 1,07 a 4,81 . Outro fator analisado foi o tempo de permanência nesta UTI. A mediana do tempo de perma- 
nência foi de 22 dias para os casos e de seis dias para os controles $(p<0,001) .^{4}$

A pneumonia que ocorre em pacientes internados em unidades de terapia intensiva determina al tas taxas de letalidade quando comparado com taxas de pacientes com pneumonia hospitalizados em outras unidades do hospital.

Diferentes microorganismos colonizam a nasofaringe e o epitélio traqueobrônquico tornando o diagnóstico etiológico difícil. Os dados coletados de hospitais americanos pelo sistema National Nosocomial Infection Surveillance (NNIS) demonstram que entre 60 a $70 \%$ das pneumonias hospitalares são causadas por bacilos aeróbios Gram-negativos, os quais são distribuídos de forma semel hante tanto em hospitais de comuni dade quanto universitários. ${ }^{5}$

Embora os bacilos Gram-negativos, Staphylococcus aureus e outras bactérias Gram-positivas sejam os patógenos mais freqüentemente identificados, também podem ser agentes causais Legionella sp.; vírus e fungos. A virulência, o tamanho do inóculo e a interação com as defesas do hospedeiro são determinantes críticos para o desenvolvimento da pneumonia.

A pesar do grande avanço tecnológico e da terapia antimicrobiana no cuidado aos pacientes graves, as taxas de letalidade dos pacientes que desenvolvem pneumonia tanto adquirida na comunidade como durante a hospitalização continuam elevadas. A terapêutica é freqüentemente empírica, baseada no dignóstico clínico e radiológico dirigida para os microorganismos mais comuns. Um regime terapêutico empírico deve considerar, para as pneumonias bacterianas comunitárias, o importante papel do Streptococcus pneumoniae, Haemophilus influenzae, Moraxella catarrhalis e Staphylococcus aureus al ém do M. pneumoniae, C. pneumoniaee L. pneumophila. Para as pneumonias adquiridas no ambiente hospitalar a terapêutica deve incluir a ação contra Klebsiella sp., Enterobacter sp., Pseudomonas aeruginosa, S. aureus e, em algumas instituições, Acinetobacter baumannii.

A cefepima é uma nova cephalosporina parenteral de amplo aspectro de atividade tanto para bactérias Gram-negativas como Gram-positivas aeróbias.

Este antimicrobiano também é ativo contra diversos microorganismos Gram-negativos resistentes para cefoxitina, ceftriaxona e cefotaxima, como também cepas de E nterobacter, Citrobacter, Klebsiella, Serratia e Providencia resistentes à ceftazidima. $8,10,14,15,16,18,19,21$ As propriedades da cefepima que determinam estas vantagens no es- pectro de atividade incluem um estrutura molecular zwiteriônica que permite rápi da penetração através da membrana externa das bactérias Gram-negativas, a estabilidade e baixa afinidade pelas beta-lactamases no espaço periplásmico ea alta afinidade para as proteínas ligadoras de penicilina. ${ }^{14,15} \mathrm{O}$ uso da cefepima está relacionado, provavelmente, a menor probabilidade de selecionar cepas resistentes e conseqüentemente alterar a microbiota dos pacientes hospitalizados quando comparado aos antimicrobianos atualmente disponíveis. ${ }^{15}$

Diversos estudos clíni cos prévios com o objetivo de avaliar a eficácia de antimicrobianos para o tratamento de pneumonias são difíceis de serem avaliados.

No presente estudo analisamos a eficácia de uma cefalosprina de quarta geração (cefepima) no tratamento de pneumonias graves tanto adquiridas na comunidade como hospitalares.

\section{CASUÍSTICA E MÉTODOS}

O estudo clínico foi desenvolvido em diversos centros médicos brasileiros, não comparativo, que envolveu 148 pacientes com idade superior a 18 anos, com o objetivo de avaliar a eficácia da cefepima no tratamento das pneumonias graves tanto comunitárias como hospitalares. Setenta e um hospitais participaram do estudo durante o período de junho a setembro de 1997. Foi definida como hospitalar, toda pneumonia desenvolvida no período superior a 72 horas após a internação por outra doença. Todos os outros casos de pneumonia foram consideradas adquiridas na comunidade. Para o diagnóstico de pneumonia adquirida na comunidade, o paciente necessariamente deveria apresentar um exame radiológico de tórax com infiltração pulmonar, sem evidências de outras causas e a presença de dois dos seguintes critérios: a) febre (temperatura axilar $>38^{\circ} \mathrm{C}$ ); b) leucocitose com mais de 10.000 células $/ \mathrm{mm}^{3}$; c) tosse produtiva com escarro purulento; d) dispnéia; e) dor torácica ventilatório dependente. A pneumonia hospitalar foi definida de acordo com um dos seguintes critérios: 1) macicez ou submacicez na percussão ou estertores creptantes ao exame clínico do tórax e mais um dos seguintes: a) aparecimento de escarro purulento ou mudança das características do escarro existente na admissão hospitalar, b) microorganismo isolado em hemocultura; c) microorganismo isolado no lavado broncoalveolar ou biópsia pulmonar; d) evidência histológica de pneumonia ou 2) exame radiológico de tórax apresentado uma nova ou progressiva in- 
filtração, consolidação, cavitação ou derrame pleural e qualquer um dos seguintes: a) aparecimento de escarro purulento ou mudança das características do escarro existente na admissão hospitalar; b) microorganismo isolado em hemocultura; c) microorganismo isolado no lavado broncoalveolar ou biópsia pulmonar; d) evidência histológica de pneumonia.

Os esquemas posológicos utilizados empregaram doses de 1.000 a $2.000 \mathrm{mg}$ a cada 8 ou 12 horas dependendo da gravidade. Em todos os esquemas foi permitido que se procedesse ajuste na dose da cefepima nos pacientes com alteração da função renal de acordo com uma tabela de correção para depuração de creatinina distribuída para os pesquisadores.

F oi permitido durante o estudo a admi nistração de antifúngicos (fluconazol ou anfotericina B). Caso houvesse suspeita de que a eti ol ogia da pneumonia fosse aspirativa, era permitido a utilização de metronidazol ou clindamicina. Foi permitido o uso de vancomicina ou aminoglicosídeo a critério do pesquisador em pacientes que apresentassem infecção grave com ou sem bacteremia.

F oram colhidas, em período não superior a 48 horas antes do iníci o do tratamento, cul turas apropriadas para o isolamento, indentificação e realização de provas de sensibilidade dos microorganismos envolvidos no processo infeccioso, segundo os critérios do $\mathrm{N}$ ational Committefor Clinical Laboratory Standard. ${ }^{17}$ A sensibilidade à cefepima das cepas bacterianas isoladas foi determinada através do método de difusão em disco.

Para as avaliações quanto a eficácia e segurança foram utilizados parâmetros clínicos e laboratoriais. As respostas bacteriológicas foram classificadas como erradicação (eliminação dos microorganismos isolados pré-terapêutica), falha (continuidade do isolamento dos mi croorganismos causadores da infecção), superinfecção (aquisi ção de um novo microorganismo que requeresse tratamento adicional durante a vigência da terapêutica em estudo) e indeterminado (não foi possível proceder a avaliação da resposta bacteriológica à cefepima). A resposta clínica à terapêutica foi avaliada de acordo com a evolução do paciente durante o estudo. A resposta clínica foi analisada pelo pesquisador responsável por cada centro até 48 horas após o término do tratamento com a cefepima. As respostas clínicas foram classificadas como resol ução (desapareci mento dos sinais e sintomas relacionados à infecção), resol ução parcial (melhora dos sinais e sintomas relacionados à infecção) e falha (diminuição insufi ciente ou piora dos sinais e sintomas relacionados à infecção, de tal forma que tenha sido necessária a introdução de terapêutica adicional ou troca da medicação).

Todos os eventos clínicos adversos ou anormalidades de exames laboratoriais ocorridas durante a vigência do tratamento ou até 48 horas do período após a interrupção do estudo foram registrados.

\section{RESULTADOS}

Setenta e um centros contribuíram com o estudo, admitindo 148 pacientes (62 pacientes com pneumonia hospitalar, 34 com pneumonia comunitária e 52 indeterminada). Os dados demográficos da população de pacientes que desenvolveram pneumonia hospitalar ou comunitária estão apresentados na tabela 1. Não houve diferença entre a distribuição por sexo tanto para os pacientes tratados para pneumonia hospitalar como para comunitária. A maioria dos pacientes nos dois grupos apresentavam doenças crônicas prévias, principalmente doença pulmonar obstrutiva crônica. No grupo de pacientes com pneumonia hospitalar, 29/62 (46,77\%) estavam em ventilação mecânica no momento do início do tratamento. A média do tempo de tratamento com cefepi ma foi de 11,54 $\pm 3,21$ dias no grupo com pneumonia hospitalar e $10,44 \pm 2,01$ no grupo com pneumonia comunitária. Na população com pneumonia comunitária houve tratamento com a cefepima associado a outros antimicrobianos em 9/34 $(26,47 \%)$ pacientes: 5 (14,70\%) ami cacina; $2(5,88 \%)$ clindamicina; $3(9,09 \%)$ metronidazol e $2(6,06 \%)$ ciprofl oxacina.

Os microorganismos isolados relacionados com a pneumonia estão apresentados na tabela 2 . As bactérias Gram-negativas estavam associadas a $78,6 \%$ nas pneumonias hospitalares e $40 \%$ nas comunitárias.

\section{DISCUSSÃO}

A tabela 3 apresenta a eficácia e os eventos adversos com o tratamento da cefepima em todas as formas de pneumonia. O índice de resolução clínica com o tratamento com cefepima foi de 107/148 $(72,29 \%)$ em todas as formas de pneumonia. N os pacientes tratados apenas com monoterapia com cefepima a resol ução clínica foi de $75 \%$. Os eventos adversos foram raros (5/148; 3,38\%) em todos os pacientes tratados. Os seguintes eventos adversos foram observados durante o tratamento com cefepima: plaquetopenia $(0,67 \%)$; diarréia $(0,67 \%)$; alteração do nível de consciência $(0,67 \%)$; reação alérgica $(0,67 \%)$ e um caso não especificado.

O tratamento empírico corretamente indicado com antimicrobianos por via parenteral continua 


\begin{tabular}{|c|c|c|}
\hline \multicolumn{3}{|c|}{$\begin{array}{l}\text { Tabela 1. Variáveis apresentadas no momento do diagnóstico de } \\
\text { pneumonia hospitalar ou comunitária na população estudada. }\end{array}$} \\
\hline Variável & $\begin{array}{l}\text { População que } \\
\text { desenvolveu } \\
\text { pneumonia } \\
\text { hospitalar }(\mathrm{N}=62)\end{array}$ & $\begin{array}{c}\text { População que } \\
\text { desenvolveu } \\
\text { pneumonia } \\
\text { comunitária ( } \mathrm{N}=34)\end{array}$ \\
\hline \multicolumn{3}{|l|}{ Sexo-N (\%) de: } \\
\hline Masculino & $32(51,61)$ & $19(55,88)$ \\
\hline Feminino & $30(48,39)$ & $15(44,12)$ \\
\hline 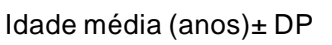 & $58,35 \pm 18,67$ & $54,52 \pm 21,96$ \\
\hline Peso médio $(\mathrm{Kg}) \pm \mathrm{DP}$ & $68,20 \pm 11,18$ & $68,41 \pm 11,73$ \\
\hline \multicolumn{3}{|l|}{$\begin{array}{l}\text { Diagnóstico } \\
\text { concomitante- } \\
\mathrm{N}(\%)^{\star}\end{array}$} \\
\hline DPOC & $21(33,87)$ & $11(32,35)$ \\
\hline Cardiopatia & $18(27,41)$ & $9(26,47)$ \\
\hline AVC & $7(11,29)$ & $0(0,0)$ \\
\hline Neoplasia & $5(8,06)$ & $3(8,82)$ \\
\hline Diabetes mellitus & $5(8,06)$ & $2(5,88)$ \\
\hline Politraumatismo & $17(27,41)$ & $2(5,88)$ \\
\hline IRC & $2(3,23)$ & $1(2,94)$ \\
\hline Infecção em outros sítios & $15(24,19)$ & $4(11,76)$ \\
\hline Outros & $44(70,96)$ & $21(61,76)$ \\
\hline \multicolumn{3}{|c|}{$\begin{array}{l}\text { Cirurgia nas últimas } 48 \text { hs. } \\
\text { do Diagnóstico }-\mathrm{N}(\%) \text { : }\end{array}$} \\
\hline Sim & $18(29,03 \%)$ & 0,0 \\
\hline Não & $44(70,97 \%)$ & $34(100 \%)$ \\
\hline \multicolumn{3}{|l|}{ N (\%) de pacientes com: } \\
\hline Ventilação assistida & $29(46,77)$ & 0,00 \\
\hline Ventilação não assistida & $33(53,23)$ & $34(100 \%)$ \\
\hline \multicolumn{3}{|l|}{$\begin{array}{l}\text { Tempo (dias) total de } \\
\text { uso da }\end{array}$} \\
\hline Cefepima: média \pm DP & $11,54 \pm 3,21$ & $10,44 \pm 2,01$ \\
\hline \multicolumn{3}{|l|}{$\begin{array}{l}\text { Terapêutica com cefepima } \\
\text { N (\%): }\end{array}$} \\
\hline Monoterapia & $29(46,77 \%)$ & $25(73,53 \%)$ \\
\hline Em associação & $33(53,23 \%)$ & $9(26,47 \%)$ \\
\hline
\end{tabular}

DPOC = doença pulmonar obstrutiva crônica; $A V C=$ acidente vascular cerebral; IRC = insuficiência renal crônica.

* Os pacientes apresentaram mais de um diagnóstico concomitante

sendo a principal medida na terapêutica de pneumonias graves. O diagnóstico eti ol ógico das pneumonias continua sendo difícil na prática clínica. Existem dificuldades técnicas para o diagnóstico rápido das pneumonias tanto as adquiridas na comunidade como nas hospitalares.

O lavado broncoalveolar ou o escovado protegido com cultura quantitativa do material obtido são os procedimentos adequados para o diagnóstico das pneumonias. Assim, poucos centros nacionais possuem um serviço de broncoscopia e um laboratório de microbiologia capaz de atender todos os casos de pneumonia.

Diversos estudos têm analisado diferentes esquemas antimicrobianos no tratamento das pneumonias, entretanto, conclusões definitivas acerca

\begin{tabular}{|lcc|}
\hline $\begin{array}{l}\text { Tabela 2. Microorganismos associados } \\
\text { população tratada com cefepima. }\end{array}$ & $\begin{array}{c}\text { a pneumonia na } \\
\text { População que } \\
\text { desenvolveu } \\
\text { pneumonia } \\
\text { hospitalar (N=62) }\end{array}$ & $\begin{array}{c}\text { População que } \\
\text { desenvolveu } \\
\text { pneumonia } \\
\text { comunitária (N=34) }\end{array}$ \\
\hline $\begin{array}{l}\text { Mseudomonas } \\
\text { aeruginosa }\end{array}$ & $5(8,06 \%)$ & $2(5,88 \%)$ \\
Pseudomonassp. & $7(11,29 \%)$ & 0,0 \\
Klebsiella sp. & $6(9,68 \%)$ & $2(5,88 \%)$ \\
Escherichia coli & $3(4,84 \%)$ & 0,00 \\
Acinetobacterbaumannii & $2(3,23 \%)$ & 0,00 \\
Enterobactersp. & $2(3,23 \%)$ & 0,00 \\
Staphylococcus aureus & $3(4,84 \%)$ & $1(2,94 \%)$ \\
Streptococcus & $3(4,84 \%)$ & $2(5,88 \%)$ \\
pneumoniae & $3(4,84 \%)$ & $1(2,94 \%)$ \\
Outros & $34 / 62(54,83 \%)$ & $8 / 34(23,52 \%)$ \\
Total & &
\end{tabular}

da eficácia ou segurança dos antimicrobianos que estavam sendo utilizados têm sido comprometida pela forma na qual os resultados são analisados e apresentados. Embora com casuística adequada, este estudo multicêntrico e prospectivo foi desenvolvido de forma aberta e não comparativa.

U ma das questões clínicas importantes referese à dúvida quanto ao uso de um único antibiótico ser suficiente para o tratamento de pneumonia bacteriana grave, especialmente naqueles casos onde a infecção tenha sido causada por bacilos Gram-negativos adquiridos durante a permanência no hospital. Desta forma, em alguns pacientes foi permitido que fosse associado à cefepi ma outro antimi crobiano dependendo da evolução clínica.

A cefepima é uma nova cefal osporina parenteral de amplo espectro de ati vidade tanto para bactérias Gram-negati vas como Gram-positivas aeróbias.

Este antimicrobiano também é ativo contra diversos microorganismos Gram-negativos resistentes para ceftriaxona e cefotaxima, como cepas de Enterobacter, Citrobacter, Serratia eProvidência resistentes à ceftazi dima. 8,10,14.15,16,19 A cefepi ma apresenta excelente concentração em mucosa brônquica e em secreção traqueobrônquica e em diversos estudos têm si do utilizada no tratamento de infecções graves incluindo pneumonias hospitalares e comunitárias graves. 6,7,9,11,12,13

E m nosso meio, Sader et al. em 1997 estudaram 982 cepas consecutivamente isoladas de 18 hospitais brasileiros durante o período de dezembro de 1995 a março de $1996 .{ }^{21} \mathrm{~A}$ atividade de cefepi ma foi superior àquela apresentada pelas outras cefalosporinas contra a maioria das espécies avaliadas. Contra enterobactérias, 96,5\% (602/624) das amos- 


\begin{tabular}{|c|c|c|c|}
\hline Variável & $\begin{array}{l}\text { Pneumonia hospitalar } \\
\qquad(\mathrm{N}=62)\end{array}$ & $\begin{array}{l}\text { Pneumonia comunitária } \\
\qquad(\mathrm{N}=34)\end{array}$ & $\begin{array}{l}\text { a Todas as pneumonias } \\
\text { (incluem as indeterminadas) } \\
\text { ( } N=148)\end{array}$ \\
\hline \multicolumn{4}{|c|}{ Resposta clínica N (\%) } \\
\hline Resolução & $42(68,85 \%)$ & $26(76,47 \%)$ & $107(72,29 \%)$ \\
\hline Resolução parcial & $14(22,95 \%)$ & $8(23,53 \%)$ & $30(20,27 \%)$ \\
\hline Falha & $5(8,20 \%)$ & 0,00 & $10(6,75 \%)$ \\
\hline Não avaliável & $1(1,61 \%)$ & 0,00 & $1(0,67 \%)$ \\
\hline \multicolumn{4}{|l|}{$\begin{array}{l}\text { Resposta clínica } \\
\text { nos pacientes } \\
\text { tratados apenas } \\
\text { com cefepima } \\
\text { (monoterapia) } \\
\text { N (\%) }\end{array}$} \\
\hline Resolução & $20(68,967)$ & $18(72,0 \%)$ & $63(75,0 \%)$ \\
\hline Resolução parcial & $8(27,59 \%)$ & $7(28,0 \%)$ & $19(22,61 \%)$ \\
\hline Falha & $1(3,54 \%)$ & $0(0,0 \%)$ & $2(2,39 \%)$ \\
\hline Não avaliável & $0(0,0 \%)$ & $0(0,0 \%)$ & $0(0,0 \%)$ \\
\hline Total de pacientes & $29(100 \%)$ & $25(100 \%)$ & $84(100 \%)$ \\
\hline \multicolumn{4}{|l|}{$\begin{array}{l}\text { Média do } \mathrm{n}^{\circ} \mathrm{de} \\
\text { Leucócitos } / \mathrm{mm}^{3} \pm \\
\text { DP }\end{array}$} \\
\hline Pré-tratamento & $17.699 \pm 7.212$ & $16.875 \pm 6.740$ & $19.723 \pm 6.726$ \\
\hline Pós-tratamento & $9.662 \pm 3.531$ & $9.060 \pm 1.877$ & $9.366 \pm 3.393$ \\
\hline \multicolumn{4}{|l|}{$\begin{array}{l}\text { Eventos adversos- } \\
\mathrm{N}(\%)\end{array}$} \\
\hline Presente & $1(1,61 \%)$ & 2 (5,88\%) & $5(3,38 \%)$ \\
\hline Ausente & $57(91,93 \%)$ & $31(91,17 \%)$ & $137(92,56 \%)$ \\
\hline Não relatado & $4(6,46 \%)$ & $1(2,95 \%)$ & $6(4,06 \%)$ \\
\hline
\end{tabular}

tras foram sensíveis à cefepima, enquanto somente $88 \%$ das amostras foram sensíveis às cefal osporinas de ter ceira geração. A superi ioridade da cefepi ma foi mais evidente contra Enterobacter cloacae, Enterobacter aerogenes e Citrobacter sp. Contra essas espécies, a atividade da cefepima foi duas a oito vezes mai or que as cefal osporinas de terceira geração. Todas as cefalosporinas testadas apresentaram boa atividade contra espécies de $E$. coli e de Proteus mirallis. A atividade contra Klebsiella pneumoniae também foi semel hante entre os antibióticos testados. Os $\mathrm{MICs}_{90}$ variaram de $8 \mathrm{mg} / \mathrm{ml}$ (cefepima, $90 \%$ de sensibilidade) a $32 \mathrm{mg} / \mathrm{ml}$ (ceftriaxona, 84\% de sensibilidade).

O papel das cefalosporinas de quarta geração no tratamento de infecções causadas por cepas produtoras de beta lactamases de espectro ampliado (ESBL) ainda não se encontra bem esclarecido. As amostras de $K$. pneumoniae também foram testadas para amicacina, ciprofloxacina e imipenem, sendo que as porcentagens de sensi bilidade foram: $76 \%, 94 \%$ e $98 \%$, respectivamente. A cefepima e a ceftazidima demonstraram atividade semel hante contra $\mathrm{P}$. aeruginosa, com discreta superioridade de cefepima. O $\mathrm{MIC}_{90}$ foi de $16 \mathrm{mg} / \mathrm{ml}$ para a cefepima e de $32 \mathrm{mg} / \mathrm{ml}$ para a ceftazidima. Todas as cefalosporinas demonstram atividade semeIhante, porém pouca atividade contra Acinetobacter sp. Neste estudo, a cefepima foi o antimicrobiano mais ativo contra estafil ococos sensíveis à oxacilina, enquanto a ceftazi dima foi o menos ativo. Cem por cento dos estafilococos sensíveis à oxacilina foram sensíveis à cefepima, $\left(\mathrm{MIC}_{90} 16 \mathrm{mg} / \mathrm{ml}\right.$ ). Todas as cefalosporinas foram bastante ativas contra Streptococcus pneumoniae e ini bi ram 100\% das amostras testadas. Porém, ceftriaxona e a cefotaxima foram as cefalosporinas mais ativas $\left(\mathrm{MIC}_{90}=0,064 \mathrm{mg} / \mathrm{ml}\right.$ ) contra essa espécie. Todas as cefal osporinas apresentaram baixa atividade contra Enterococcus faecalis. Assim, estes dados microbiológicos demonstraram que as cefal osporinas de quarta geração representam uma melhor opção que as cefalosporinas de terceira geração no tratamento de infecções graves por Citrobacter freundii, E nterobacter sp. e Serratia marcescens em hospitais nos quais essas espécies apresentam altos índices de resistência à cefalosporinas ${ }^{21}$.

Entretanto, é importante enfatizar que as cefalosporinas de quarta geração não apresentam boa atividade contra alguns microorganismos sa- 
bidamente resistentes aos antimicrobianos da classe das cefalosporinas, como, por exemplo, estafi lococos resistentes à oxacilina, enterococos e Stenotrophomonas (Xanthomonas) maltophilia. Outro microorganismo freqüentemente resistente às cefalosporinas é o Acinetobacter baumannii.

E m nosso estudo, o índice de resolução clínica com o tratamento com a cefepima foi de 107/148 (72,29\%), em todas as formas de pneumonia. Nos pacientes tratados apenas com cefepima (monoterapia) a resolução clínica foi de 20/29 (68,97\%) nos pacientes com pneumonia hospitalar e 18/25 (72\%) naqueles com pneumonia adquirida na comunidade. A anál ise da resposta microbiológica foi comprometida, pois em um grande número de pacientes não foi possível a coleta de material pré e/ou pós tratamento. Fink et al, em 1994, avaliando os índices de resposta clínica em estudo comparativo utilizando ci profloxacina versus imi penem no tratamento de pneumonia grave encontram resposta clínica de $69 \%$ no grupo ci profl oxacina e $56 \%$ no grupo imipenem. ${ }^{20}$

E ventos adversos foram raros (5/148; 3,38\%) em todos os pacientes tratados. Os seguintes eventos adversos foram observados durante o tratamento com cefepima: plaquetopenia $(0,67 \%)$; diarréia $(0,67 \%)$; alteração do nível de consciência $(0,67 \%)$; reação alégica $(0,67 \%)$ e um caso não especificado.

Este estudo clínico multicêntrico prospectivo, aberto e não comparativo, demonstrou que a cefepima é uma boa opção no tratamento de pneumonias hospitalares e comunitárias graves, com perfil de segurança adequado, uma vez que foram raros os relatos de eventos adversos.

\section{AGRADECIMENTOS}

Aos seguintes médicos que participaram deste estudo: Aldindo Edilcio Massucato, Alexandre Maia Lage, Antonio Carlos Machado, Benedito Aparecido Caiel, Caio Marcelo Ferrari, Claudio Flamarion Ribeiro dos Santos, Claudio Luiz Lemos de M orais, Concetta Esposito, Denise Machado Medeiros, Edilson Madureira Reis, Eduardo Calixto Salida, E uzébio Silvio J odar Lopes, Flávio Monteiro Bardos Maciel, Francisco Gilberto Vasconcelos Monteiro, Gilberto Barbosa, Guido A. Pena, I van Lorenzato, J ander Silva e Castro, J oão Geraldo Houli, J oaquim Pereira da Silva, J orge Anunciação, J osé Carlos Ribeiro, J osé Eduardo Cançado, J osé Oliveira Calvete, J oseu Reinaldo Ferreira, Kilma Mattos, Leandro da Silva Santos, Luciele Oliveira Shifelbain, Luis A. M. Chiarelli, Luiz Carlos Goulart, Luiz Eduardo Schein, Luiz Felipe Kugler Mendes, Luiz Gonzaga Massari
Filho, Luiz Ricardo Ataide Castro, Marco Antonio Liserre Garcia, Marcos Antonio Vieira Senra, Marcos Cesar Valerio de Almeida, Margarida Neves, Maria Cristina Arnaldo Caprini, Maria da Glória P. Fernandes, Onesimo Domingo F. Filho, Paulo Athaide, Renato Chiavassa, Renato Maciel, Rita de Cássia Lacerda de Paula, Roberto Stirbulov, Rosário do Socoro M ota Silva, Rubens Antonio Bento Ribeiro, Sergio R. Savytsky e Wilson Roberto Oliver.

\section{SUMMARY}

\section{Treatment of nosocomial pneumonia: a pros- pective and multicenter study used cefepi me}

OвJ ECTIVE. To evaluate efficacy and safety of cefepime in severe pneumonia of hospitalized patients.

Design and Patients. A prospective, multicenter, open trial was performed with 148 patients (62 patients with nosocomial pneumonia; 34 with community-acquired pneumonia and 52 undefined forms). Cefepime was intravenously administer ed (1,000 to 2,000mg every 12 hours), and doses were adjusted for renal function. The efficacy endpoint was clinical response at 48 hours after completion of therapy.

Results. The mean age was $56.4 \pm 20,31$ years. The most common bacterias isolated from patients with nosocomial pneumonia were: 5 (8.06\%) Pseudomonas aeruginosa; 7 (11.29\%) Pseudomonas sp.; 6 (9.68\%) Klebsiella sp.; 3 (4.84\%) E.coli; 2 (3.23\%) Acinetobacter baumannii; 3 (4.84\%) Staphyl ococcus aureus; 3 (4.84\%) Streptococcus pneumoniae; 5 (8.06\%) others. The most common isolates from patients with community-acquired pneumonia were: 2 (5.88\%) Streptococcus pneumoniae; 1 (2.94\%) S. aureus; 2 (5.88\%) P. aeruginosa and $2(5.88 \%) \mathrm{K}$. pneumoniae. Clinical efficacy was demonstrated in $137 / 148(92.56 \%)$ of the cases since improvement was obtained in $20.27 \%$ and healing in $72.29 \%$. Failure of the treatment was observed in 10 patients (6.75\%) and one patient the evaluation was not possible. Adverse events were reported for 5/ 148 patients (3.38\%).

Conclusion. Our data suggest that cefepi me was safe and effective for treatment of severe pneumonia in hospitalized patients. [Rev Ass Med Brasil 1999; 45(1): 2-8.]

KEY WORDS: Community acquired pneumonia. Nosocomial pneumonia. Cephalosporin. Cefepime.

\section{REFERÊNCIAS BIBLIOGRÁFICAS}

1. Marrie, T.J .; Durant, H.; Yates, L. Community-acquired pneumonia requiring hospitazation: five-year prospective study. Rev. Infect. Dis., 1989; 11:586-599. 
2. Torres, A.; Serra-Batlles, J .; Ferrer, A et al. SevereCommunityacquireds pneumonia. E pidemi ol ogy and prognosticfactors. Am. Rev. Respir.Dis.; 1991; 144:312-318.

3. Austrian, R \& Gold, J . Pneumococcal bacteremia with especial referencetobacteremic pneumococcal pneumonia. Ann. Intern. Med., 1964; 60:759-776

4. Medeiros, E.A.S. - Efeito da pneumonia hospitalar sobre a letal idade e o tempo de hospital ização em adul tos internados em unidadedeterapia intensiva. São Paulo, 1991. 131p. Tese de Mestrado - Escola Paulista de Medicina.

5. Centers F or Disease Control and Prevention - Draft guideline for prevention of nosocomial pneumonia; Notice of Comment Period, 1994, 59:4980-5022.

6. Bosso J A, Saxon BA, Matsen J M: Comparative activity of cefepime alone and in combination against clinical isolates Pseudomonas aeruginosa and cepacia from cystic fibrosis pactients. Antimicrob Agent Chemother 1991, 35:783.

7. Chadha C, Wise R, Baldwin DR et al: Cefepime concentrations in bronchial mucosa and serum following a single 2 gran intravenous dose. J . Antimi crobial Chemother, 1990, 25:959.

8. Durval J , Soussy C.J , Acar J .F, et al: In vitro antibacterial activity of cefepime: A multicentre study. J Antimicrob Chermother, 1993, 32(suppl B): 55.

9. Giamarellou H: Low dosage cefepime treatment for serious bacterial infection. J Antimicrob Chemother, 1993, 32(suppl B): 123.

10. Gillium J .G, Polk R.E: Focus on cefepime a fourth generation, extended-spectrum cephal osporin. Hosp F ormulary, 1994, 29:503.

11. J auregui $L, M$ atzke D, Scott $M$, et al: Cefepime as treatment for osteomyelitis and other severe bacterial infectious. J Antimicrob Chemother, 1993, 32(suppl B): 141.

12. KovarikJ .M, Ter J C, Rademaker CMA, etal: Phammacokinetics of cefepimein patients with respiratory tract infections. Antimicrob agents Chemother, 1990, 34: 1885.

13. Mccabe R, Chirugi V, Farkas A.S, et al: Cefepime in the treatment of lower respiratory tract infections. Am J Med 1993, 95(suppl 4 A): 24.

14. Neu H.C: Safety of cefepime: A new extended-spectrum cephal osporin antibiotic. AmJ Med 95 1993, (suppl 4 A): 67.

15. Barradell L.B \& Bryson, H.M. Cefepime. A review of its antibacterial activity, pharmacokinetic properties and therapeutic use. Drugs, 1994, 47:471-505.

16. Frei R, J ones R.N, Pignatari A.C et al. Antimicrobial /activity of FK-037, a new broad-spectrum cephal osporin. I nternational in vitro comparison with cefepime and ceftazidime. Diagn Microbial I nfect Dis, 1994, 18:167-173.

17. National Commite For Clinical Laboratory Standards (NCCLS). Performance standards for antimicrobial dis suscepti bility tests: approved standard. $5^{\text {th }}$ ed. NCCLS document M2-A5. Vilanova, Pennsylvania: National Committefor Clinical Laboratory Standards, 1993.

18. Martínez-Martínez L, Hernández-Alléz S, Albertí S et al. In vivo selection of porin-deficient mutants of Klebsiella pneumoniae with increased resistance to cefoxitin and extendedspectrum cephal osporins. Antimicrob Agents chemother, 1996 40:342-348.

19. Gales A.C, Sader H.S, Machado Amo, et al. Comparação das atividades antimi crobianas das cefepima e da ceftazi dima em 1015 amostras bacterianas bacterians isol adas em São Paulo. J Bras Patol, 1995, 31:55-60.

20. Fink, M.P; Snydman, D.R; Niederman, M.S et al. Treatment of severe pneumonia in hospitalized patients: results of a multicenter, randomized, double-blind trial comparing intravenous ciprofloxacin with imipenem-cilastatin. Antimicrob Agents Chemother, 1994, 38:547-557.

21. Sader, H.S; Minica, I; Rossi, F ; Zoccoli, C; Montelli, A; Sampaio, J ; Segura, A; Magalhães, M; Nowakonski, A; Mendes, C.M. Atividade in vitro da cefepima em comparação com outras cefal osporinas deampl oespectro contra amostras clínicas de 18 hospitais brasileiros. Arqs Bras deMed, 1996 70:123-127. 